\title{
Plasmonic Refractive Index Sensors Based on One- and Two-Dimensional Gold Grating on a Gold Film
}

\author{
Jiankai ZHU ${ }^{1}$, Xiangxian $\mathrm{WANG}^{1 *}$, Yuan $\mathrm{WU}^{1}$, Yingwen $\mathrm{SU}^{1}$, Tianxu JIA ${ }^{1}$, \\ Hua YANG ${ }^{1}$, Liping ZHANG ${ }^{1}$, Yunping $\mathrm{QI}^{2}$, and Xiaolei $\mathrm{WEN}^{3}$ \\ ${ }^{1}$ School of Science, Lanzhou University of Technology, Lanzhou 730050, China \\ ${ }^{2}$ College of Physics and Electronic Engineering, Northwest Normal University, Lanzhou 730070, China \\ ${ }^{3}$ Center for Micro- and Nanoscale Research and Fabrication, University of Science and Technology of China, Hefei \\ 230026, China \\ ${ }^{*}$ Corresponding author: Xiangxian WANG \\ E-mail: wangxx869@lut.edu.cn
}

\begin{abstract}
In this paper, we propose two kinds of composite structures based on the one- and two-dimensional (1D\&2D) gold grating on a gold film for plasmonic refractive index sensing. The resonance modes and sensing characteristics of the composite structures are numerically simulated by the finite-difference time-domain method. The composite structure of the 1D gold semi-cylinder grating and gold film is analyzed first, and the optimized parameters of the grating period are obtained. The sensitivity and figure of merit (FOM) can reach $660 \mathrm{RIU} / \mathrm{nm}$ and $169 \mathrm{RIU}^{-1}$, respectively. Then, we replace the 1D grating with the 2D gold semi-sphere particles array and find that the 2D grating composite structure can excite strong surface plasmon resonance intensity in a wider period range. The sensitivity and FOM of the improved composite structure can reach $985 \mathrm{RIU} / \mathrm{nm}$ and $298 \mathrm{RIU}^{-1}$, respectively. At last, the comparison results of the sensing performance of the two structures are discussed. The proposed structures can be used for bio-chemical refractive index sensing.
\end{abstract}

Keywords: Surface plasmon; refractive index; sensor; grating

Citation: Jiankai ZHU, Xiangxian WANG, Yuan WU, Yingwen SU, Tianxu JIA, Hua YANG, et al.,"Plasmonic Refractive Index Sensors Based on One- and Two-Dimensional Gold Grating on a Gold Film," Photonic Sensors, 2020, 10(4): 375-386.

\section{Introduction}

Due to the continuous development of the fabrication technology in the sub-wavelength scale, various nano-devices based on surface plasmons have been developed rapidly [1-3], which have achieved many breakthroughs from theory to reality. For example, based on the fact that the propagating surface plasmons can break through the diffraction limit, a more miniaturized and integrated waveguide device can be made $[4,5]$. The electric field enhancement [6-8] and strong absorption [9, 10] caused by the local surface plasmons are widely used in surface-enhanced Raman scattering [11-13] and enhanced absorption [14, 15]. In addition, the unique optical properties of surface plasmons have a wide range of applications in photocatalysis [16-20], absorber [21-24], photolithography [25-28], filter $[29,30]$, optical data storage [31, 32], and other fields [33-36]. At present, one of the most important researches focusing on surface plasmons is

Received: 12 February 2020 / Revised: 28 June 2020

(C) The Author(s) 2020. This article is published with open access at Springerlink.com

DOI: $10.1007 / \mathrm{s} 13320-020-0598-\mathrm{x}$

Article type: Regular 
refractive index sensing [37-42]. The resonance wavelength or angle of surface plasmons is extraordinarily sensitive to environmental changes and the range of surface plasmon effect can be controlled at the nano-scale, which results in a considerable application future in the development of high sensitivity, high integration, and portable refractive index sensors.

The prism excited [43-45] plasmonic refractive index sensors have limited their further development owing to their bulky volume and expensive cost. In recent years, the refractive index sensor based on the surface plasmon excited via the array of the nano-grating [46-49] provides an effective scheme for the realization of low-cost, portable, and integrated sensors, and is attracting more and more attention. Under the optimized geometric parameters, the diffraction caused by the nano-grating can compensate for the appropriate optical momentum to achieve the effective coupling between the incident light and the surface plasmons, thereby exciting the strongly propagating surface plasmon resonances. In addition, the nano-grating in the sensing structure can be either dielectric or metallic [50-52], and the metal nano-grating can generally excite more surface plasmon resonance modes. When surface plasmon resonances are used for refractive index sensing, propagating surface plasmons have more advantages than local surface plasmons. This is caused by the characteristics of the propagating surface plasmon. A wider range of interaction between the propagating surface plasmons and the environment can lead to a higher sensitivity. The resonance peak excited by the propagating surface plasmons usually has a narrower full width at half maximum (FWHM), which means a higher figure of merit (FOM) and a refractive index resolution. In the literature, there have been many reports on surface plasmon refractive index sensors excited by one-dimensional (1D) gratings [50-52].
It is significant to explain the forming reason and characteristics of surface plasmon resonances before a micro-nano structure is proposed for plasmonic refractive index sensing. In this paper, we use the finite-difference time-domain method to obtain the reflection spectra and electric field distributions of the composite structures, and then analyze the resonance modes and sensing characteristics of the surface plasmon. We have studied the composite structures of different dimensions of the gold gratings coupled with the gold films and comparatively explain the characteristics of the surface plasmon resonance excited by gratings via expanding the dimension of gratings. We find that although the fabrication cost of the two-dimensional (2D) grating will be increased relatively, the improvement advantage brought by the $2 \mathrm{D}$ grating is also very obvious. The sensitivity, FOM, and sensing range of the 2D grating composite structure are improved, and the polarization direction of the incident light does not need to be along the direction of the period. Finally, sensing performance parameters of $1 \mathrm{D}$ and $2 \mathrm{D}$ gratings composite structures are compared, highlighting the advantages of the $2 \mathrm{D}$ grating composite structure used in the refractive index sensing.

\section{1D grating composite structure}

\subsection{Structure design and analysis method}

Figure 1 shows the three-dimensional (3D) geometry structure of the composite structure of the 1D gold semi-cylinder grating and the gold film, and the illustration in the figure is the schematic diagram of the side plane of the composite structure. From top to bottom, the structures are the gold semi-cylinder grating, silicon dioxide thin-film, gold film, and silicon dioxide substrate. As shown in the figure, the red line shows the grating radius of the gold semi-cylinder unit, and the black line shows the period of the 1D grating. Here, we fix the radius of 
the gold semi-cylinder and the thickness of the gold film to $60 \mathrm{~nm}$ and $150 \mathrm{~nm}$, respectively, with an adjustable period.

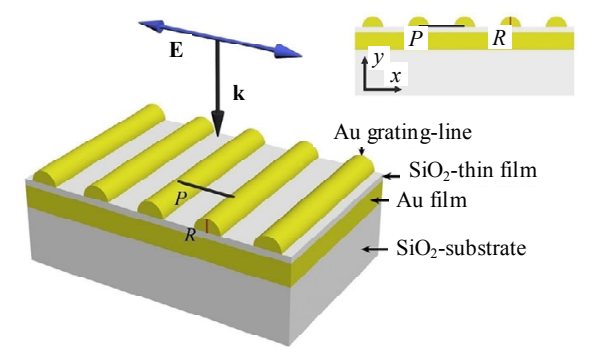

Fig. $13 \mathrm{D}$ geometric structure of the 1D grating composite structure. From top to bottom, the components consist of the 1D gold semi-cylinder grating, silicon dioxide thin film, gold film, and silicon dioxide substrate (the black arrow indicates the direction of propagation of the incident light, the blue arrow indicates the polarization direction, and the black and red lines are the period and radius, respectively).

The surface plasmons of the composite structure are excited vertically by the linearly polarized light in the direction of polarization along the periodic direction, as shown by the black and blue arrows in Fig. 1. One period has been selected as the calculation unit during the simulation since the structure is periodic along the $x$ direction. The grid sizes in the $x$ and $y$ directions are chosen to be $2 \mathrm{~nm} \times 2 \mathrm{~nm}$. The periodic boundary conditions and absorbing boundary conditions are set in the $x$ and $y$ directions of the calculation element, respectively. The dielectric constants of the gold in the simulation are selected from the experimental data supported by the Drude model [53].

\subsection{Theoretical analysis of resonance modes of the composite structure}

In the case of the TM polarized wave incident vertically, the expression of the surface plasmon resonance excited by the $1 \mathrm{D}$ grating is given as [54]

$$
\lambda_{\text {res }}=\frac{P}{m} \sqrt{\frac{\operatorname{Re}\left(\varepsilon_{\text {metal }}\right) \cdot n_{\text {analyte }}^{2}}{\operatorname{Re}\left(\varepsilon_{\text {metal }}\right)+n_{\text {analyte }}^{2}}}
$$

where $m$ is the diffraction order of the 1D grating and is an integer, $P$ is the period of the 1D grating, $n_{\text {analyt }}$ is the refractive index (RI) of the analyte, and $\varepsilon_{\text {metal }}$ is the complex dielectric constant of the gold. The resonance wavelength of the surface plasmon excited by the grating is related to the period. The resonance wavelength moves to the longer wavelength with an increase in the period, and the larger period means that the higher order can be excited. It should be noted that when the electric vector is parallel to the $1 \mathrm{D}$ grating (TE polarization), the surface plasmons cannot be excited since the free electrons cannot effectively couple the incident light energy in this direction to form an oscillation.

The refractive index of the analyte is set as 1.0, and the simulation results of the reflection spectra of the 1D grating composite structure under different periods are shown in Fig. 2, with the period varying from $400 \mathrm{~nm}$ to $1400 \mathrm{~nm}$. Colors represent the distributions of reflection intensity, as shown in the color bar. It can be seen from the simulation results that the 1D grating composite structure mainly excites two kinds of surface plasmon resonance modes, Modes 1 and 2. When the period is greater than $900 \mathrm{~nm}$, the third mode (Mode 3) can be excited. As shown by the white dotted line in the figure, Modes 1 and 3 show the linear sensitivity of the resonance wavelength to the period. Combined with the grating equation, it is concluded that they are excited by the optical momentum compensated via the $1 \mathrm{D}$ grating with different diffraction orders. It is noted that Modes 1 and 2 repel each other when the resonance wavelength is close. Similar phenomena appeared in the previous report [55]. When the period is greater than $900 \mathrm{~nm}$, the resonance wavelength of Mode 2 hardly changes with the period, and the reason for the red shift of Mode 2 when the period is less than $900 \mathrm{~nm}$ can be attributed to the hybridization of Modes 1 and 2. In order to clearly compare the theoretical analysis and simulation results, we mark the resonance wavelengths (black stars) calculated via (1) in Fig. 2. Here, we calculate the resonance wavelengths from $600 \mathrm{~nm}$ to $1300 \mathrm{~nm}$ in steps of $100 \mathrm{~nm}$. It can be observed that the surface plasmon excitation equation of the $1 \mathrm{D}$ grating is in good agreement with the simulation results. The differences between 
simulation results and theoretical analysis about Mode 1 mainly come from the hybridization of Modes 1 and 2.

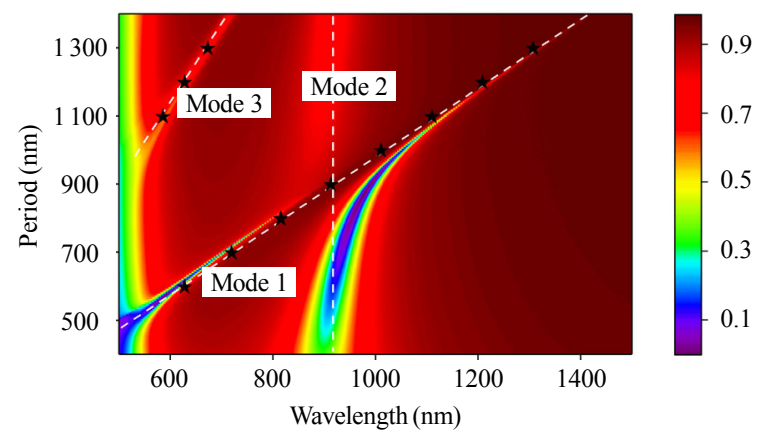

Fig. 2 Reflective spectra of the 1D grating composite structure. The period ranges from $400 \mathrm{~nm}$ to $1400 \mathrm{~nm}$ and the colors represent the reflectivity distributions. The black stars show the resonance wavelengths calculated via the theoretical analysis under different periods.

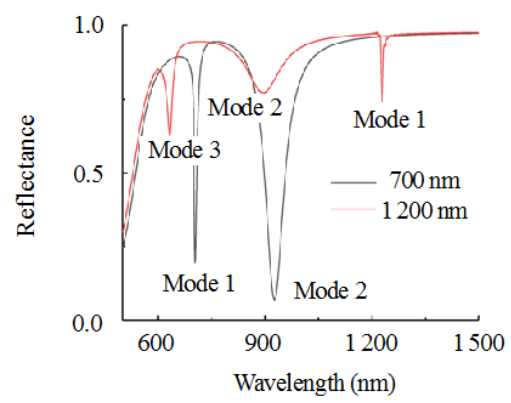

(a)

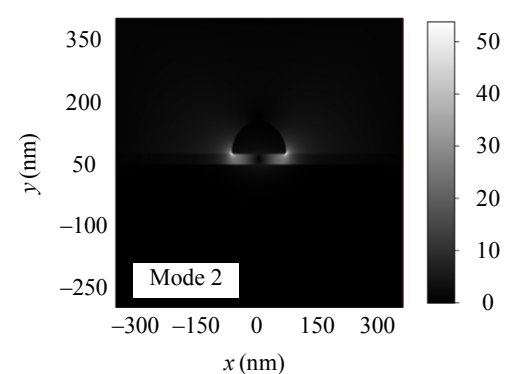

(c)

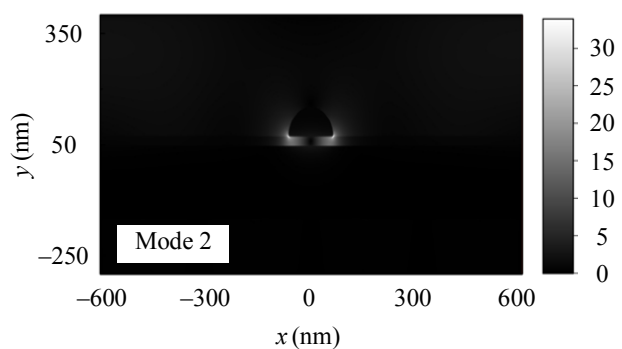

(e)
In view of the intuitiveness of the electric field distribution, it is necessary to give the electric field distributions under the resonance wavelengths when interpreting the surface plasmon resonance. In order to better illustrate the resonance characteristics of each mode in combination with the electric field distribution, we separately give the reflection spectra with periods of $700 \mathrm{~nm}$ and $1200 \mathrm{~nm}$ (data from Fig. 2), and label each mode as shown in Fig.3(a). It can be seen from Fig. 3(a) that when the period is $700 \mathrm{~nm}$, the composite structure can excite two strong resonance modes, Modes 1 and 2. When the period is $1200 \mathrm{~nm}$, Mode 1 redshifts to a longer wavelength, and the resonance wavelength of Mode 2 slightly changes. Mode 3 corresponds to the diffraction order $m=2$ under the large period.

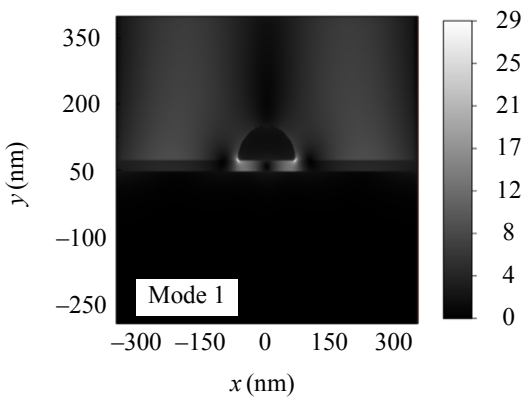

(b)



(d)



(f)

Fig. 3 Plasmonic responses of the 1D grating composite structure: (a) reflection spectra of the 1D grating composite structure with periods of $700 \mathrm{~nm}$ and $1200 \mathrm{~nm}$, respectively. Electric field distribution $|\mathbf{E}| /\left|\mathbf{E}_{0}\right|$ at the resonance wavelength of (b) Mode 1 and (c) Mode 2 with a period of $700 \mathrm{~nm}$, and at the resonance wavelength of (d) Mode 3, (e) Mode 2, and (f) Mode 1 with a period of $1200 \mathrm{~nm}$. 
Figures 3(b) and 3(c) show the electric field distributions of the two modes with a period of $700 \mathrm{~nm}$, respectively. The electric field distribution of Fig. 3(b) shows the diffraction characteristics of the nano-gratings, which clearly shows that Mode 1 is excited by the gratings. The electric field enhancement of Fig.3(c) is mainly localized on both sides of the gold semi-cylinder grating, which explains the reason why the resonance wavelength of Mode 2 is not sensitive to the period. Figures3(d) (Mode 3), 3(e) (Mode 2), and 3(f) (Mode 1) show the electric field distributions in three resonance modes with a period of $1200 \mathrm{~nm}$. The electric field distribution of Fig. 3(f) is similar to that in Fig.3(b), which demonstrates that the two modes are indeed the same mode excited via the $1 \mathrm{D}$ grating under different periods with the same diffraction order $(n=1)$. The similar conclusion can be drawn by comparing Figs. 3(c) and 3(e). Through the comparison of Figs. 3(d) and 3(f), it can be found that both modes are caused by the diffraction of the gold semi-cylinder grating, and the obvious different electric field distributions between the two modes can be attributed to that they are two resonance modes with different diffraction orders. It is worth noting that although more resonant modes can be excited with an increase in the period, the resonance intensity will be weakened. This can be seen from an increase in the reflectivity in the reflection spectrum of Fig. 3(a).

\subsection{Sensing performance of the composite structure}

Sensitivity and FOM are often used to describe the performance of the refractive index sensor. The higher the two parameters are, the better the performance of the sensor is. The refractive index sensitivity is defined as $S=\Delta \lambda / \Delta n[56,57]$, where $\Delta n$ refers to the change of the refractive index, and $\Delta \lambda$ refers to the corresponding deviation of the resonance wavelength under the change of the refractive index $\triangle n$. The $F O M$ is defined as $F O M=$
$S / F W H M$, where $F W H M$ refers to the full width at half maximum of the resonance peak used for refractive index sensing.

In view of the fact that the refractive index sensing needs higher resonance intensity of surface plasmons, we expect to use more resonance modes for refractive index sensing at the same time. Therefore, according to the reflection spectra in different periods shown in Fig. 2, we choose the period equal to $700 \mathrm{~nm}$ as our optimization parameter. Figure 4 shows the reflection spectra of the $1 \mathrm{D}$ grating composite structure under different refractive indices of the analyte. The first six curves have refractive index steps of 0.02 , and then the last four curves have refractive index steps of 0.1 . The simulation range of refractive indices is set from 1.0 to 1.4 , which basically covers the refractive index of the analyte being gas or part of the liquid. For example, the alcohol solution or sugar solution has refractive indices related to the corresponding mixing proportion. As shown in Fig. 4, it is obvious that the resonance wavelengths of Modes 1 and 2 move to the longer wavelength with an increase in refractive indices and the linear relationship is good. It can also be observed that, although Mode 1 is no longer suitable for refractive index sensing due to the weakening of the resonance intensity when the refractive index is greater than 1.1, the repulsion between Modes 1 and 2 has successfully reduced the FWHM of Mode 2, thus increasing its FOM.

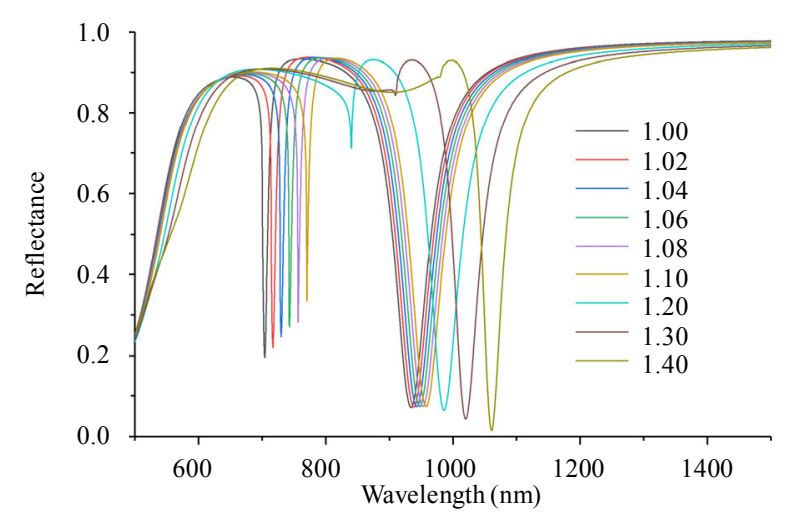

Fig. 4 Reflective spectra of the composite structure. The analyte refractive index ranges from 1.0 to 1.4 (the first six curves have refractive index steps of 0.02 , and then the last four curves have refractive index steps of 0.1 ). 
In order to describe the sensing performance of the 1D grating composite structure in detail, we evaluate the relationship between the resonance wavelengths and $F O M$ with refractive indices in two modes. Figure 5(a) shows the sensitivity curve and FOM change of Mode 1 in the refractive index sensing range of 1.0 to 1.1 . It can be observed that the resonance wavelengths have a good linear relationship with the change of refractive indices, and FOM increases nonlinearly with the change of refractive indices. Depending on the calculation formula of sensitivity, the slope of the straight line is

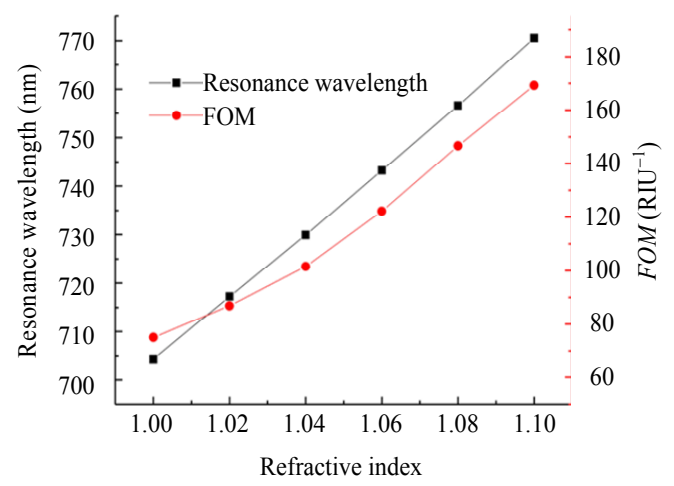

(a) the sensitivity. The sensitivity of Mode 1 is $660 \mathrm{RIU} / \mathrm{nm}$, and the highest FOM can reach $169 \mathrm{RIU}^{-1}$. Figure 5(b) shows the sensitivity curve and FOM change of Mode 2 in the refractive index range of 1.0 to 1.4. The sensitivity of Mode 2 is $316 \mathrm{RIU} / \mathrm{nm}$ by fitting the relationship between the resonance wavelengths and the refractive indices. It is worth noting that FOMs of both modes increase as the refractive index increases. This can be attributed to the fact that the resonance wavelengths of the two modes approach with an increase in refractive indices, and the repulsive effect leads to a decrease in FWHM.

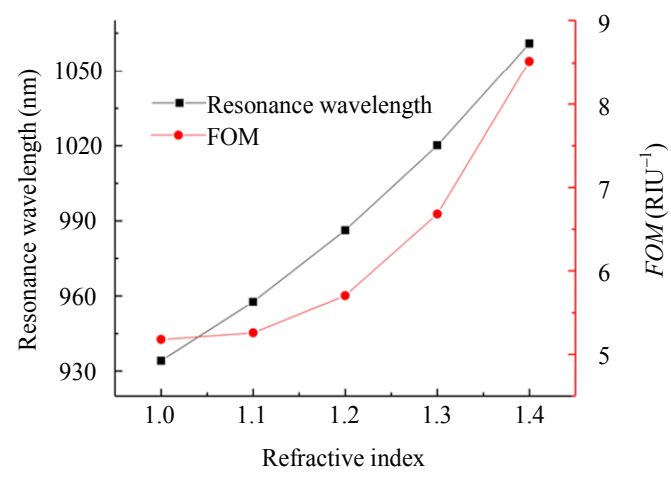

(b)

Fig. 5 Relationship of the resonance wavelength and FOM with the refractive index of the analyte in (a) Mode 1 and (b) Mode 2.

\section{2D grating composite structure}

\subsection{Structure design and resonance analysis}

Built on the study of the 1D grating composite structure, we extend the dimension of the gratings to $2 \mathrm{D}$. Figure 6 is a schematic diagram of the 2D grating composite structure. Compared with the 1D grating composite structure shown in Fig. 1, it can be seen that the 1D gold semi-cylinder grating is replaced by the 2D gold semi-sphere particles array (2D grating). Here, the radius of the gold semi-sphere is set at $160 \mathrm{~nm}$. The surface plasmon resonances are still excited by the vertical incident light with the polarization direction along the periodic direction. Obviously, the 2D grating composite structure has no requirement for the polarization direction of the incident light. In other words, the $2 \mathrm{D}$ grating composite structure can be excited by TM or TE polarization. The 2D grating composite structure is periodic along the $x$ and $y$ directions, so we select a period as the unit of calculation. The boundaries around the calculated unit are used as the periodic boundary conditions. Absorption boundary conditions are employed in the analyte and $\mathrm{SiO}_{2}$ substrate, which are the upper and lower surfaces in the actual calculation unit.

After correction of diffraction orders, the expression of surface plasmon resonances excited by the 2D grating can be expressed as $[54,55]$

$$
\lambda_{\text {res }}=\frac{P}{\sqrt{n^{2}+m^{2}}} \sqrt{\frac{\operatorname{Re}\left(\varepsilon_{\text {metal }}\right) \cdot n_{\text {analyte }}^{2}}{\operatorname{Re}\left(\varepsilon_{\text {metal }}\right)+n_{\text {analyte }}^{2}}}
$$

where $n$ and $m$ are integers, and $P$ is the period of the $2 \mathrm{D}$ grating. Figure 7 shows the reflection spectrum of the 2D grating composite structure when the scanning wavelength is from $500 \mathrm{~nm}$ to $2000 \mathrm{~nm}$ and the period changes from $800 \mathrm{~nm}$ to $1400 \mathrm{~nm}$. Compared with Fig.2, it is clear that the 
2D grating composite structure can excite stronger surface plasmon resonances in a larger period range. This is an advantage for the structure to be used in refractive index sensing, which means there are no strict geometric parameter requirements for the structure. Similar to the 1D grating composite structure, the 2D grating composite structure also excites more surface plasmon resonance modes when the period is large. As shown in Fig. 7, the resonance wavelengths of Modes 1 and 3 vary with the period, which is caused by the diffraction of the 2D gratings, corresponding to the diffraction orders $(m, n)=(1,0)$ and $(1,1)$. Mode 2 shows a relatively wide $F W H M$, and its resonance wavelength hardly changes with the period. Thus, the excitation reason can be attributed to the local surface plasmons in the particles of the gold semi-sphere. Figure 7 also shows the comparison between the resonance wavelength obtained via (2) and the simulation results. Here, we calculate the resonance wavelengths (black stars) from $900 \mathrm{~nm}$ to $1200 \mathrm{~nm}$ in steps of $100 \mathrm{~nm}$. One can note that the simulation results are almost identical with the theoretical analysis.

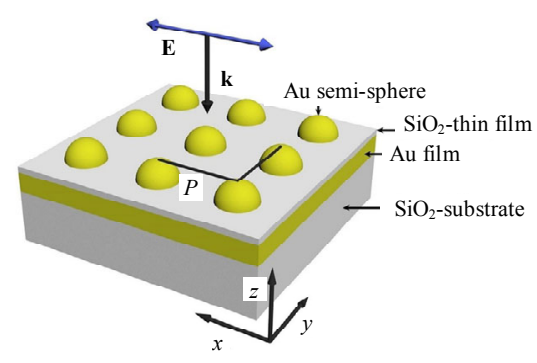

Fig. $63 \mathrm{D}$ geometric structure of the 2D grating composite structure. From top to bottom, the components consist of the 2D gold semi-sphere particles array, silicon dioxide thin film, gold film, and silicon dioxide substrate (the black arrow indicates the direction of propagation of the incident light, the blue arrow indicates the polarization direction, and the black line shows the period).

When the period is $1000 \mathrm{~nm}$, the reflection spectrum of the $2 \mathrm{D}$ grating composite structure is shown in Fig. 8(a). The main excitation modes of the composite structure are Modes 1 and 2, respectively, and the reflectivity of Mode 2 is almost zero. The electric field distributions of the two modes are shown in Figs. 8(b) and 8(c). Figure 8(b) shows the electric field distribution on the upper surface of the gold film at the resonance wavelength of Mode 1. It can be seen that the electric field enhancement is mostly on the upper surface of the gold film. This is the result that the incident light is coupled to form a surface plasmon propagating along the upper surface of the gold film. Figure 8(c) shows the electric field distribution on the lower surface of the gold semi-sphere particle at the resonance wavelength of Mode 2. The strong local electric field enhancement at both ends of the gold semi-sphere particles along the polarization direction can be observed. This is the product that the incident light is coupled to form a localized surface plasmon in the gold semi-sphere particles. It is also the reason that the resonance wavelength of Mode 2 is not sensitive to the period.



Fig. 7 Reflective spectra of the 2D grating composite structure. The period ranges from $800 \mathrm{~nm}$ to $1400 \mathrm{~nm}$ and the color represents the reflectivity distribution. The black stars show the resonance wavelengths calculated via theoretical analysis under different periods.

\subsection{Sensing performance of the composite structure}

Considering that we expect to obtain the considerable sensing range and sensitivity at the same time, we select the $2 \mathrm{D}$ grating period equal to $1000 \mathrm{~nm}$ as our optimization parameter. The reflection spectra of the $2 \mathrm{D}$ grating composite structure under different refractive indices of the analyte are illustrated in Fig. 9(a). Here, the variation range of the refractive index of the analyte is set 
from 1.0 to 1.4 in steps of 0.1 . The linear red shift of the resonance wavelength of the two modes with an increase in the refractive index can be clearly observed. Figures 9(b) and 9(c) show the relationship between the resonance wavelengths and FOM with analyte refractive indices in two modes, respectively. The sensitivities of Modes 1 and 2 are $985 \mathrm{RIU} / \mathrm{nm}$ and $332 \mathrm{RIU} / \mathrm{nm}$, respectively. The highest FOM of Mode 1 is $298 \mathrm{RIU}^{-1}$, while that of Mode 2 is almost invariant. FOM of Mode 2 is only about $3.5 \mathrm{RIU}^{-1}$ because of its wide FWHM. It

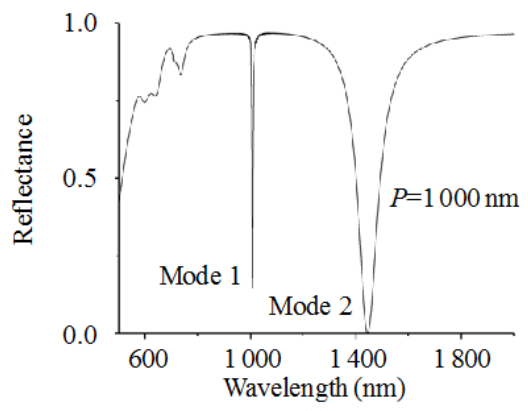

(a) should be noted that Mode 2 is caused by the coupling resonance between the oscillation frequency of the free electron in the gold particle and the incident light frequency. Such resonances will occur in a wide band of the incident light, thus showing a wide $F W H M$ in the reflection spectrum. However, the propagating surface plasmons caused by the $2 \mathrm{D}$ grating compensating optical momentum require a specific frequency of the incident light, resulting in a narrow $F W H M$ of Mode 1 in the reflection spectrum.

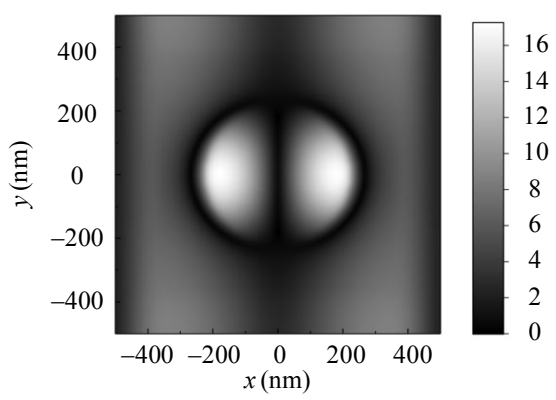

(b)



(c)

Fig. 8 Plasmonic responses of the 2D grating composite structure: (a) reflection spectrum of the 2D grating composite structure with a period of $1000 \mathrm{~nm}$, electric field distribution $|\mathbf{E}| /\left|\mathbf{E}_{0}\right|$ at the resonance wavelengths of (b) Mode 1 and (c) Mode 2.

It can also be observed from Fig. 9(a) that an increase in the refractive index will inevitably lead to the repulsion between the two modes due to the different slopes of the resonance wavelength of Modes 1 and 2 with the change of the refractive index, so that Mode 1 is no longer suitable for sensing. It can be seen from Fig. 7 that a smaller period means a shorter resonance wavelength of Mode 1, which means a larger refractive index sensing range. Therefore, the period of the geometric optimization parameter here equal to
$1000 \mathrm{~nm}$ is considered for Mode 1. In particular, it should be noted that the period of the $2 \mathrm{D}$ grating is not the smaller the better, and the resonance intensity of Mode 1 should be taken into account. In fact, because Mode 1 is caused by the surface plasmon propagating along the surface of the gold film, the larger period has a longer propagation distance. In a sense, an increase in the period will increase the range of interaction between the surface plasmon and the analyte on the structure, thus improving the refractive index sensitivity of Mode 1. 
For example, when the $2 \mathrm{D}$ grating period is equal to $1200 \mathrm{~nm}$, the sensing range of Mode 1 will be reduced to $1.00-1.16$, but the sensitivity will be increased to $1180 \mathrm{RIU} / \mathrm{nm}$.

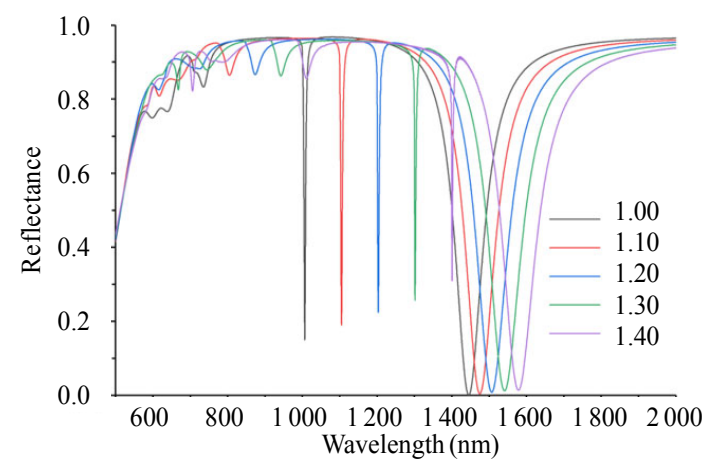

(a)

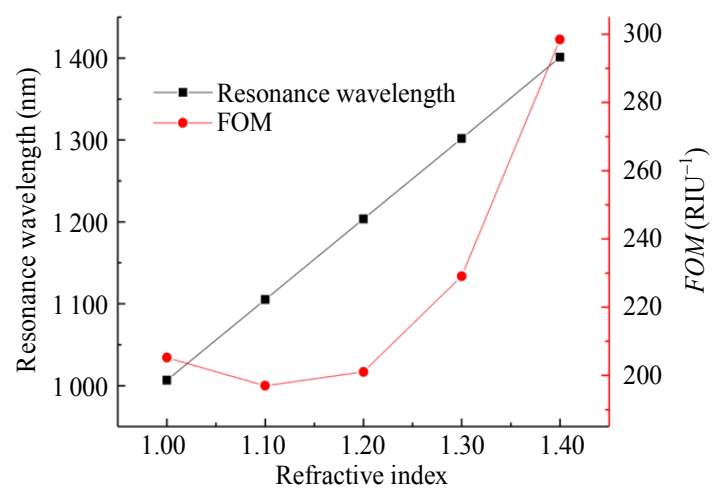

(b)

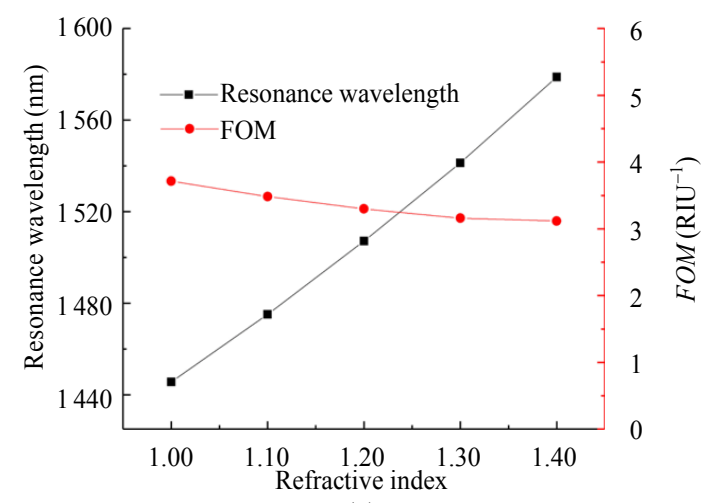

(c)

Fig. 9 Sensing performance of the 2D grating composite structure: (a) reflective spectra of the composite structure when the analyte refractive index ranges from 1.0 to 1.4 in steps of 0.1 . The relationship of the resonance wavelength and FOM with the analyte refractive index in (b) Mode 1 and (c) Mode 2.

\section{Comparison between one- and two-dimensional gratings}

The sensing parameters of the 1D grating composite structure and the $2 \mathrm{D}$ grating composite structure are shown in Table 1. The sensitivity, the maximum FOM, and the sensing range of the two modes under the two composite structures are given in the table. Compared with the $1 \mathrm{D}$ grating, Mode 1 excited by the $2 \mathrm{D}$ grating can significantly improve the performance parameters of the sensor. The main reason is that the $2 \mathrm{D}$ grating has a wider period optimization range, thus we can use a larger period. Although extending the grating dimension to $2 \mathrm{D}$ does not apparently increase the sensitivity of Mode 2 , it significantly increases the resonance intensity of Mode 2. It can be seen from the electric field distribution that the electric field enhancement of Mode 2 of the 2D grating can reach 85 times of the incident light [Fig. 3(c)], while that of the 1D grating is 53 times [Fig. 8(c)]. This is mainly due to the fact that the gold semi-sphere particles of the $2 \mathrm{D}$ grating can better excite the strong local surface plasmons than the gold semi-cylinder of the 1D grating. It is worth mentioning that although the $2 \mathrm{D}$ grating composite structure has better sensing performance and no requirements for the incident light polarization, the $1 \mathrm{D}$ grating still has the advantage of relatively simple fabrication.

Table 1 Comparison of sensing parameters (sensitivity, maximum FOM, and sensing range) between the $1 \mathrm{D}$ grating composite structure and the $2 \mathrm{D}$ grating composite structure.

\begin{tabular}{ccc}
\hline & 1D grating & 2D grating \\
\hline Mode 1: $S \backslash \max F O M \backslash$ & $660 \mathrm{RIU} / \mathrm{nm} \backslash 169 \mathrm{RIU}^{-1} \backslash$ & $985 \mathrm{RIU} / \mathrm{nm} \backslash 298 \mathrm{RIU}^{-1} \backslash$ \\
sensing range & $1.0-1.1$ & $1.0-1.4$ \\
Mode 2: $S \backslash \max F O M \backslash$ & $316 \mathrm{RIU} / \mathrm{nm} \backslash 8.5 \mathrm{RIU}^{-1} \backslash$ & $332 \mathrm{RIU} / \mathrm{nm} \backslash 3.7 \mathrm{RIU}^{-1} \backslash$ \\
sensing range & $1.0-1.4$ & $1.0-1.4$ \\
\hline
\end{tabular}

The analysis of all modes shows that Mode 1 under the 2D grating composite structure has the best refractive index sensing characteristics. Its sensitivity and FOM are higher than those reported recently [46-50]. Although some plasmonic refractive index sensors based on the waveguide structure have higher sensitivity [58, 59], the composite structures of the gold gratings and gold films have advantages in FOM since it can effectively reduce $F W H M$ of the resonance peak. 


\section{Conclusions}

In conclusion, we have studied the refractive index sensing performance of the composite structures between different dimensions of the gold gratings and gold films. It is found that not only the polarization direction of the incident light is not required by expanding the grating dimension, but also the resonance intensity of the surface plasmons is improved significantly, thus obtaining a wider period range of the composite structure for refractive index sensing. When the period of the $2 \mathrm{D}$ gratings is $1000 \mathrm{~nm}$, the sensitivity, the maximum FOM, and the refractive index sensing range can reach $985 \mathrm{RIU} / \mathrm{nm}, 298 \mathrm{RIU}^{-1}$, and 1.0 to 1.4 , respectively. When a large sensing range is not required, the sensitivity can still be improved by increasing the period appropriately. Moreover, although the sensing performance of the $2 \mathrm{D}$ grating composite structure is better than that of the 1D grating composite structure, the 1D grating with a relatively simple fabrication process is still an optional choice when the sensing sensitivity requirements are not high. The proposed composite structures are good references for the future development of the plasmonic refractive index sensor.

\section{Acknowledgment}

This work was supported by the National Natural Science Foundation of China (NSFC) (Grant No. 61865008) and the HongLiu First-Class Disciplines Development Program of Lanzhou University of Technology.

Open Access This article is distributed under the terms of the Creative Commons Attribution 4.0 International License (http://creativecommons.org/licenses/by/4.0/), which permits unrestricted use, distribution, and reproduction in any medium, provided you give appropriate credit to the original author(s) and the source, provide a link to the Creative Commons license, and indicate if changes were made.

\section{References}

[1] A. Paliwal, A. Sharma, M. Tomar, and V. Gupta,
"Long range surface plasmon resonance (LRSPR) based highly sensitive refractive index sensor using Kretschmann prism coupling arrangement," AIP Conference Proceedings, 2016, 1724(1): 020132-1-020132-5.

[2] J. You, Y. Takahashi, K. Leonard, H. Yonemura, and S. Yamada, "Influence of space arrangement of silver nanoparticles in organic photoelectric conversion devices," Journal of Photochemistry and Photobiology, A: Chemistry, 2017, 332: 586-594.

[3] Z. Wei, Z. Zhou, Q. Li, J. Xue, A. D. Falco, Z. Yang, et al., "Flexible nanowire cluster as a wearable colorimetric humidity sensor," Small, 2017, 13(27): 1700109-1-1700109-7.

[4] Y. Zhu, C. Deng, L. Huang, G. Hu, B. Yun, R. Zhang, et al., "Hybrid plasmonic graphene modulator with buried silicon waveguide," Optics Communications, 2020, 456: 124559-1-124559-6.

[5] K. Zheng, Y. Yuan, L. Zao, Y. Chen, F. Zhang, J. Son, et al., "Ultra-compact, low-loss terahertz waveguide based on graphene plasmonic technology," 2D Materials, 2020, 7(1): 015016-1-015016-8.

[6] J. Chen, X. Wang, F. Tang, X. Ye, L. Yang, and Y. Zhang, "Substrates for surface-enhanced Raman spectroscopy based on TiN plasmonic antennas and waveguide platforms," Results in Physics, 2020, 16: 102867-1-102867-6.

[7] X. Wang, Y. Wu, X. Wen, J. Zhu, X. Bai, Y. Qi, et al., "Surface plasmons and SERS application of $\mathrm{Au}$ nanodisk array and $\mathrm{Au}$ thin film composite structure," Optical and Quantum Electronics, 2020, 52: 238-1-238-11.

[8] Y. Wu, X. Wang, X. Wen, J. Zhu, X. Bai, T. Jia, et al., "Surface-enhanced Raman scattering based on hybrid surface plasmon excited by Au nanodisk and Au film coupling structure," Physics Letters A, 2020, 384(23): 126544-1-126544-6.

[9] Y. Wang, F. Qin, Z. Yi, X. Chen, Z. Zhou, H. Yang, et al., "Effect of slit width on surface plasmon resonance," Results in Physics, 2019, 15: 102711-1-102711-3.

[10] Y. Wang, Z. Chen, D. Xu, Z. Yi, X. Chen, J. Chen, et al., "Triple-band perfect metamaterial absorber with good operating angle polarization tolerance based on split ring arrays," Results in Physics, 2020, 16: 102951-1-102951-6.

[11] G. Liu, M. Yu, Z. Liu, X. Liu, S. Huang, P. Pan, et al., "One-process fabrication of metal hierarchical nanostructures with rich nanogaps for highly-sensitive surface-enhanced Raman scattering," Nanotechnology, 2015, 26(18): 185702-1-185702-9.

[12] L. Tang, Y. Liu, G. Liu, Q. Chen, Y. Li, L. Shi, et al., "A novel SERS substrate platform: spatially stacking plasmonic hotspots films," Nanoscale Research Letters, 2019, 14(1): 94-1-94-11. 
[13] X. Wang, J. Zhu, Y. Wu, Y. Xu, Y. Su, L. Zhang, et al., "Hybrid surface plasmon effect and SERS characterization in a heterogeneous composite structure of Au nano-array and Ag film," Results in Physics, 2020, 17: 103175-1-103175-5.

[14] G. Liu, J. Chen, P. Pan, and Z. Liu, "Hybrid metal-semiconductor meta-surface based photo-electronic perfect absorber," IEEE Journal of Selected Topics in Quantum Electronics, 2018, 25(3): 4600507-1-4600507-8.

[15] P. Yu, X. Chen, Z. Yi, Y. Tang, H. Yang, Z. Zhou, et al., "A numerical research of wideband solar absorber based on refractory metal from visible to near infrared," Optical Materials, 2019, 97: 109400-1-109400-6.

[16] Y. Yan, H. Yang, Z. Yi, and T. Xian, " $\mathrm{NaBH}_{4}$-reduction induced evolution of $\mathrm{Bi}$ nanoparticles from $\mathrm{BiOCl}$ nanoplates and construction of promising $\mathrm{Bi} @ \mathrm{BiOCl}$ hybrid photocatalysts," Catalysts, 2019, 9(10): 795-1-795-20.

[17] Y. Wang, H. Yang, X. Sun, H. Zhang, and T. Xian, "Preparation and photocatalytic application of ternary $n-\mathrm{BaTiO}_{3} / \mathrm{Ag} / \mathrm{p}-\mathrm{AgBr} \quad$ heterostructured photocatalysts for dye degradation," Materials Research Bulletin, 2020, 124: 110754-1-110754-10.

[18] S. Wang, H. Yang, Z. Yi, and X. Wang, "Enhanced photocatalytic performance by hybridization of $\mathrm{Bi}_{2} \mathrm{WO}_{6}$ nanoparticles with honeycomb-like porous carbon skeleton," Journal of Environmental Management, 2019, 248: 109341-1-109341-10.

[19] S. Guan, H. Yang, X. Sun, and T. Xian, "Preparation and promising application of novel $\mathrm{LaFeO}_{3} / \mathrm{BiOBr}$ heterojunction photocatalysts for photocatalytic and photo-Fenton removal of dyes," Optical Materials, 2020, 100: 109644-1-109644-11.

[20] Y. Yan, H. Yang, Z. Yi, T. Xian, and X. Wang, "Direct Z-scheme $\mathrm{CaTiO}_{3} @ \mathrm{BiOBr}$ composite photocatalysts with enhanced photodegradation of dyes," Environmental Science and Pollution Research, 2019, 26: 29020-29031.

[21] Z. Liu, P. Tang, X. Liu, Z. Yi, G. Liu, Y. Wang, et al., "Truncated titanium/semiconductor cones for wide-band solar absorbers," Nanotechnology, 2019, 30(30): 305203-1-305203-10.

[22] C. Cen, Y. Zhang, X. Chen, H. Yang, Z. Yi, W. Yao, et al., "A dual-band metamaterial absorber for graphene surface plasmon resonance at terahertz frequency," Physica E: Low-dimensional Systems and Nanostructures, 2020, 117: 113840-1-113840-8.

[23] Y. Qi, C. Liu, B. Hu, X. Deng, and X. Wang, "Tunable plasmonic absorber in THz-band range based on graphene "arrow" shaped metamaterial," Results in Physics, 2019, 15: 102777-1-102777-7.

[24] F. Qin, Z. Chen, X. Chen, Z. Yi, W. Yao, T. Duan, et al., "A tunable triple-band near-infrared metamaterial absorber based on $\mathrm{Au}$ nano-cuboids array," Nanomaterials, 2020, 10(2): 207-1-207-11.

[25] H. Tong, Y. Xu, Y. Su, and X. Wang, “Theoretical study for fabricating elliptical subwavelength nanohole arrays by higher-order waveguide-mode interference," Results in Physics, 2019, 14: 102460-1-102460-5.

[26] X. Wang, H. Tong, Z. Pang, J. Zhu, X. Wu, H. Yang, et al., "Theoretical realization of three-dimensional nanolattice structure fabrication based on high-order waveguide-mode interference and sample rotation," Optical and Quantum Electronics, 2019, 51: 38-1-38-8.

[27] X. Wang, Z. Pang, H. Yang, and Y. Qi, “Theoretical study of subwavelength circular grating fabrication based on continuously exposed surface plasmon interference lithography," Results in Physics, 2019, 14: 102446-1-102446-3.

[28] Z. Pang, H. Tong, X. Wu, J. Zhu, X. Wang, H. Yang, et al., "Theoretical study of multiexposure zeroth-order waveguide mode interference lithography," Optical and Quantum Electronics, 2018, 50: 335-1-335-9.

[29] B. Yan, A. Wang, E. Liu, W. Tan, J. Xie, R. Ge, et al., "Polarization filtering in the visible wavelength range using surface plasmon resonance and a sunflower-type photonic quasi-crystal fiber," Journal of Physics D: Applied Physics, 2018, 51(15): 155105-1-155105-7.

[30] Y. Qi, P. Zhou, T. Zhang, X. Zhang, Y. Wang, C. Liu, et al., "Theoretical study of a multichannel plasmonic waveguide notch filter with double-sided nanodisk and two slot cavities," Results in Physics, 2019, 14: 102506-1-102506-8.

[31] Y. Cui, I. Phang, R. Hegde, Y. Lee, and X. Ling, "Plasmonic silver nanowire structures for two-dimensional multiple-digit molecular data storage application," ACS Photonics, 2014, 1(7): 631-637.

[32] Q. Dai, M. Ouyang, W. Yuan, J. Li, B. Guo, S. Lan, et al., "Encoding random hot spots of a volume gold nanorod assembly for ultralow energy memory," Advanced Materials, 2017, 29(35): 1701918-1-1701918-8.

[33] T. Wu, Y. Liu, Z. Yu, H. Ye, Y. Peng, C. Shu, et al., "A nanometeric temperature sensor based on plasmonic waveguide with an ethanol-sealed rectangular cavity," Optics Communications, 2015, 339: $1-6$.

[34] G. Gao, W. Feng, W. Su, S. Wang, L. Chen, M. Li, et al., "Preparation and modification of MIL-101(Cr) metal organic framework and its application in lithium-sulfur batteries," International Journal of Electrochemical Science, 2020, 15: 1426-1436.

[35] X. Yang, Y. Lu, B. Liu, and J. Yao, "Simultaneous measurement of refractive index and temperature based on SPR in D-shaped MOF," Applied Optics, 
2017, 56(15): 4369-4374.

[36] M. Li, W. Feng, W. Su, and X. Wang, "Complex hollow structures of Cobalt(II) sulfide as a cathode for lithium-sulfur batteries," International Journal of Electrochemical Science, 2020, 15: 526-534.

[37] X. Wang, J. Zhu, X. Wen, X. Wu, Y. Wu, Y. Su, et al., "Wide range refractive index sensor based on a coupled structure of Au nanocubes and Au film," Optical Materials Express, 2019, 9(7): 3079-3088.

[38] X. Wang, J. Zhu, H. Tong, X. Yang, X. Wu, Z. Pang, et al., "A theoretical study of a plasmonic sensor comprising a gold nano-disk array on gold film with a $\mathrm{SiO}_{2}$ spacer," Chinese Physics B, 2019, 28(4): 044201-1-044201-6.

[39] C. Liu, W. Su, F. Wang, X. Li, L. Yang, T. Sun, et al., "Theoretical assessment of a highly sensitive photonic crystal fibre based on surface plasmon resonance sensor operating in the near-infrared wavelength," Journal of Modern Optics, 2019, 66(1): $1-6$.

[40] Q. Liu, B. Yan, and J. Liu, "U-shaped photonic quasi-crystal fiber sensor with high sensitivity based on surface plasmon resonance," Applied Physics Express, 2019, 12(5): 052014-1-052014-4.

[41] C. Li, B. Yan, and J. Liu, "Refractive index sensing characteristics in a D-shaped photonic quasi-crystal fiber sensor based on surface plasmon resonance," Journal of the Optical Society of America A, 2019, 36(10): 1663-1668.

[42] Y. Qi, Y. Wang, X. Zhang, C. Liu, B. Hu, Y. Bai, et al., "A theoretical study of optically enhanced transmission characteristics of subwavelength metal Y-shaped arrays and its application on refractive index sensor," Results in Physics, 2019, 15: 102495-1-102495-6.

[43] X. Wang, X. Wu, J. Zhu, Z. Pang, H. Yang, and Y. Qi, "Theoretical investigation of a highly sensitive refractive-index sensor based on $\mathrm{TM}_{0}$ waveguide mode resonance excited in an asymmetric metal-cladding dielectric waveguide structure," Sensors (Switzerland), 2019, 19(5): 1187-1-1187-10.

[44] D. M. Hernandez, J. S. Velazquez-Gonzalez, D. Luna-Moreno, M. Torres-Cisneros, and I. Hernandez-Romano. "Prism-based surface plasmon resonance for dual-parameter sensing," IEEE Sensors Journal, 2018, 18(10): 4030-4037.

[45] A. Paliwal, M. Tomar, and V. Gupta, "Refractive index sensor using long-range surface plasmon resonance with prism coupler," Plasmonics, 2019, 14(2): 375-381.

[46] J. Chen, H. Nie, C. Peng, S. Qi, C. Tang, Y. Zhang, et al., "Enhancing the magnetic plasmon resonance of three-dimensional optical metamaterials via strong coupling for high-sensitivity sensing," Journal of Lightwave Technology, 2018, 36(16): 3481-3485.
[47] J. Chen, W. Fan, T. Zhang, X. Chen, J. Wu, D. Li, et al., "Engineering the magnetic plasmon resonances of metamaterials for high-quality sensing," Optics Express, 2017, 25(4): 3675-3681.

[48] J. Chen, J. Yuan, Q. Zhang, H. Ge, C. Tang, Y. Liu, et al., "Dielectric waveguide-enhanced localized surface plasmon resonance refractive index sensing," Optical Materials Express, 2018, 8(2): 342-347.

[49] J. Chen, W. Fan, P. Mao, C. Tang, Y. Liu, Y. Yu, et al., "Tailoring plasmon lifetime in suspended nanoantenna arrays for high-performance plasmon sensing," Plasmonics, 2017, 12(3): 529-534.

[50] M. Abutoama and I. Abdulhalim, "Self-referenced biosensor based on thin dielectric grating combined with thin metal film," Optics Express, 2015, 23(22): 28667-28682.

[51] M. Abutoama and I. Abdulhalim, "Angular and intensity modes self-referenced refractive index sensor based on thin dielectric grating combined with thin metal film," IEEE Journal of Selected Topics in Quantum Electronics, 2017, 23(2): 4600309-1-4600309-9.

[52] Z. Zhang, L. Wang, H. Hu, K. Li, X. Ma, and G. Song, "A high figure of merit localized surface plasmon sensor based on a gold nanograting on the top of a gold planar film," Chinese Physics B, 2013, 22(10): 104213-1-104213-4.

[53] P. B. Johnson and R. W. Christy, "Optical constants of the nobel metals," Physical Review B, 1972, 6(12): 4370-4379.

[54] J. Cao, Y. Sun, Y. Kong, and W. Qian, "The sensitivity of grating-based SPR sensors with wavelength interrogation," Sensors (Switzerland), 2019, 19(2): 405-1-405-9.

[55] Y. Chu and K. B. Crozier, "Experimental study of the interaction between localized and propagating surface plasmons," Optics Letters, 2009, 34(3): 244-246.

[56] C. Liu, L. Yang, X. Lu, Q. Liu, F. Wang, J. Lv, et al., "Mid-infrared surface plasmon resonance sensor based on photonic crystal fibers," Optics Express, 2017, 25(13): 14227-14237.

[57] C. Liu, L. Yang, Q. Liu, F. Wang, Z. Sun, T. Sun, et al., "Analysis of a surface plasmon resonance probe based on photonic crystal fibers for low refractive index detection," Plasmonics, 2018, 13(3): 779-784.

[58] X. Jin, X. Huang, J. Tao, X. Lin, and Q. Zhang, “A novel nanometeric plasmonic refractive index sensor," IEEE Transactions on Nanotechnology, 2010, 9(2): 134-137.

[59] S. Zou, F. Wang, R. Liang, L. Xiao, and M. Hu, "A nanoscale refractive index sensor based on asymmetric plasmonic waveguide with a ring resonator: a review," IEEE Sensors Journal, 2015, 15(2): 646-650. 